\title{
Utilization of 3D Printing in Replacement of Basic Plastic Workpieces
}

\author{
Álmos KRISTÁLY1 ${ }^{1}$, PÉTER FICZERE 2 \\ ${ }^{1}$ BME Department of Railway Vehicles and Vehicle System Analysis, H-1111 Budapest, Stoczek str. 2. St bld., \\ Hungary \\ 2 BME Department of Railway Vehicles and Vehicle System Analysis, H-1111 Budapest, Stoczek str. 2. St bld., \\ Hungary, 0000-0003-3207-5501 \\ Corresponding author e-mail: ficzere.peter@kjk.bme.hu
}

In the experiment, a 3D printed cogwheel is made using the FDM technology to replace a broken part in a sewing machine. The aim of the project is to examine if a 3D model can be created and manufactured using only entry-level technical knowledge and tools. By the end of the article, it will be apparent that creating functioning plastic parts with a hobby $3 D$ printer and basic CAD experience is very much possible.

Keywords: 3D printing, gear, sewing machine, additive manufacturing, reverse engineering

\section{Introduction}

Additive manufacturing is an invention of the 20th century [1], which fundamentally changed production technology as we know it. It is an ever-growing sector, flourishing today.

In contrast with the traditional subtractive manufacturing processes, during which material is removed from an overall-dimensioned workpiece using cutting machines such as lathes, milling machines, drills etc. until the desired geometry is achieved, additive manufacturing (implied by the name) is an additive process, during which the final workpiece is created from an existing model, point-cloud, or occasionally mould.

The essence of the FDM process is loading a plastic (seldom metal) wire into a 3D printing machine [2], where the material is heated to and melted on a pre-set temperature. The melt is distributed on a bed according to the desired geometry, which cools down and solidifies. This way, a thin layer is created, on top of which another one can be made and so on, until the complete spatial piece is done [3].

The aforementioned plastics differ based on the target application (and technological limits of the machine), from everyday materials such as polyamide and nylon to special and extraordinary ones which offer high rigidity, creep strength, or rubber-like amorphous qualities.

A 3D model is "sliced" into hundreds, or even thousands of layers along a base plane by a software. These segments are "printed" layer by layer on top of each other, where they solidify and fuse together [4]. Finally, the complete geometry is achieved. This technology opens opportunities to produce parts which would be physically impossible to machine, for example enclosed holes, sockets, inner geometries. 
Naturally, the FDM method has boundaries as well: printing "in air" is impossible, a certain layer must have at least partial contact with a lower layer to hold it in place during the printing. If this requirement cannot be fulfilled on a part or parts of the desired geometry, so called "supports" are implemented into the model. This results in excess material, which is wasted, since it needs to be removed at the end of production.

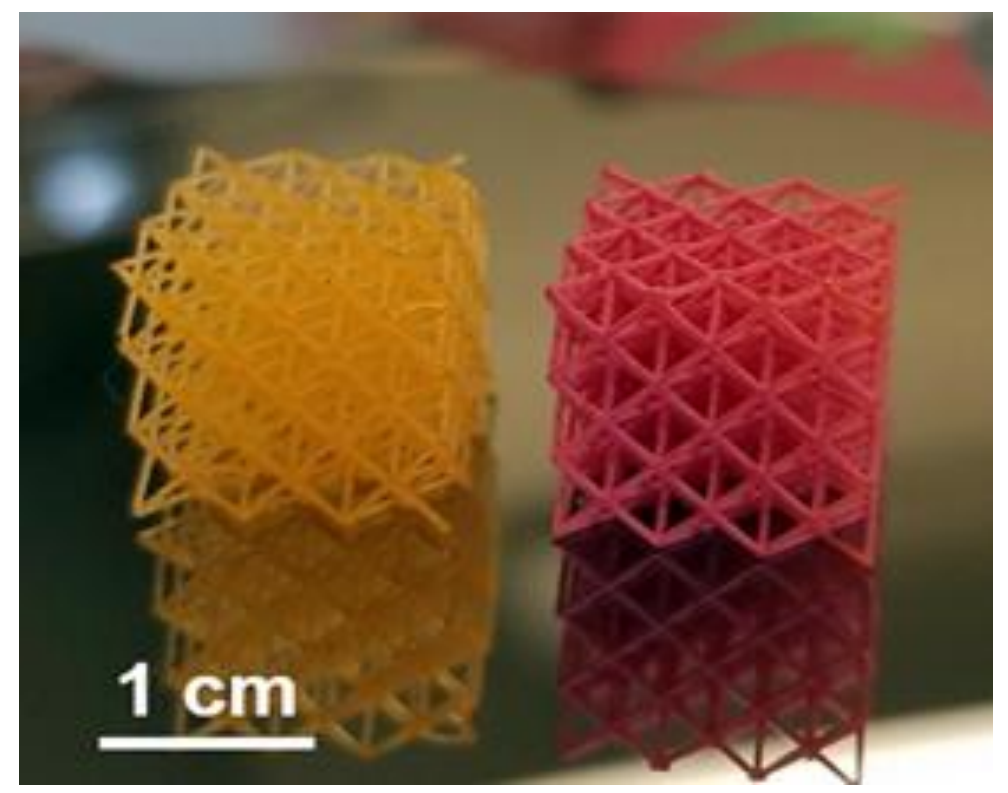

Figure 1. Cubes like this are nearly impossible to produce with traditional methods [5]

Weaknesses of 3D printing include low surface quality (generally, although it is better than cast or forged pieces), anisotropy, and slow production. The aforementioned layering strongly affects the bearing capacity of the products. Consequently, a pulled axle layered perpendicular to its load will break significantly sooner than the same axle layered parallel to the load. 


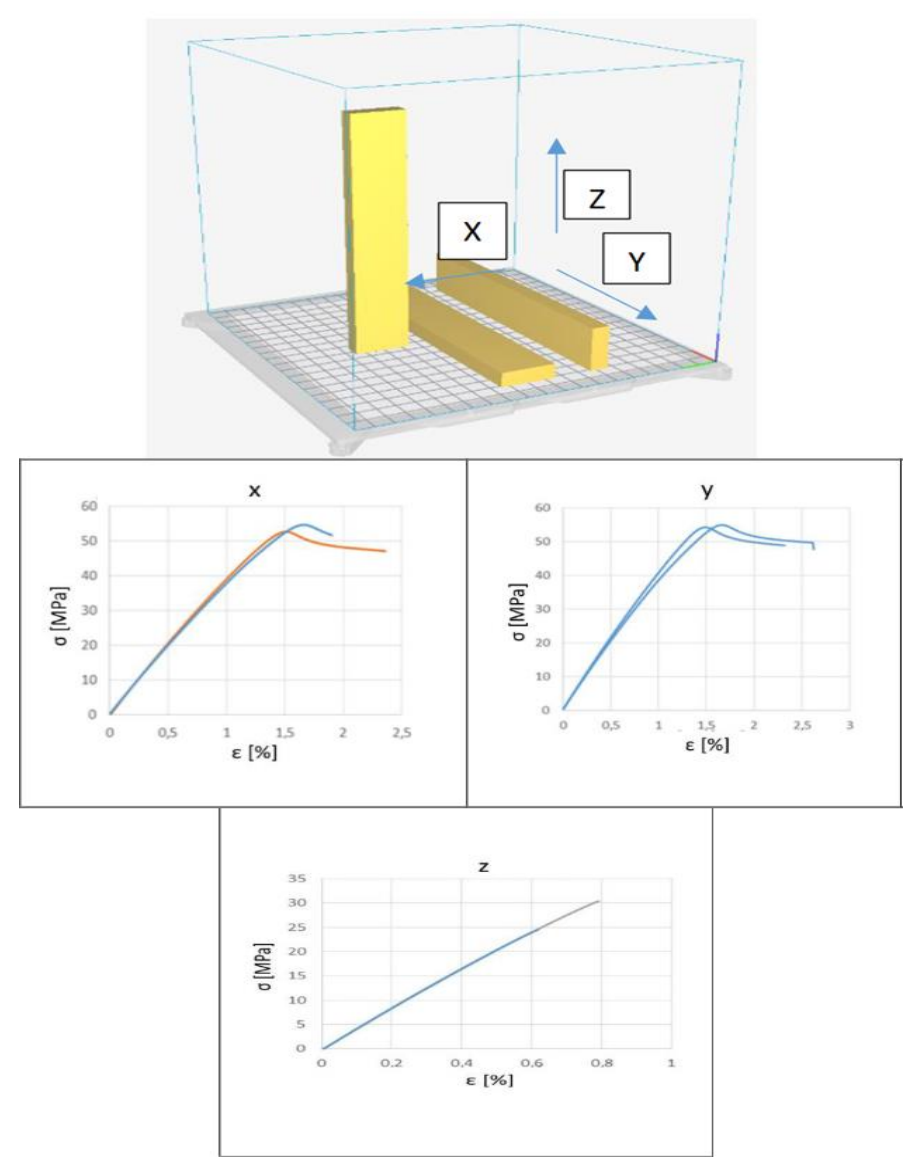

Figure 2. Stress-strain diagrams of plastic tensile specimen printed in different directions [6]

Its greatest advantage lies in its cost efficiency, especially while producing custom parts or small batches, in which case starting up or reconstructing a whole production line would be a huge waste of resources. It is a single-step, scrap- and wasteless procedure, which requires little to no post-processing, making it a highly cost-efficient and space saving method. 3D printers for home use can be purchased for the cost of a low-end personal computer, enabling the average person to create smaller parts or trinkets without using complex, expensive technologies and technicians.

By combining 3D printing with other technologies, such as the also new-found reverse engineering, during which the models and parameters required for reconstruction are created from an existing workpiece, new opportunities arise. Old or rare parts found in vintage cars, electric appliances, watches for example, that cannot be acquired by regular means anymore can be produced again relatively cheap and easy.

\section{Experimental}

With that in mind, let me present the central subject of my documentation. I will attempt to model and replace a sewing machine's broken plastic gear with a 3D printed part. The part is responsible for the reciprocating motion of the needle during zig-zag stitching, during which the needle moves forward 
while alternating between the left and right side of the stitch, as seen in fig. 3. It is mostly used for finishing a seam, making it an indispensable function of a sewing machine.

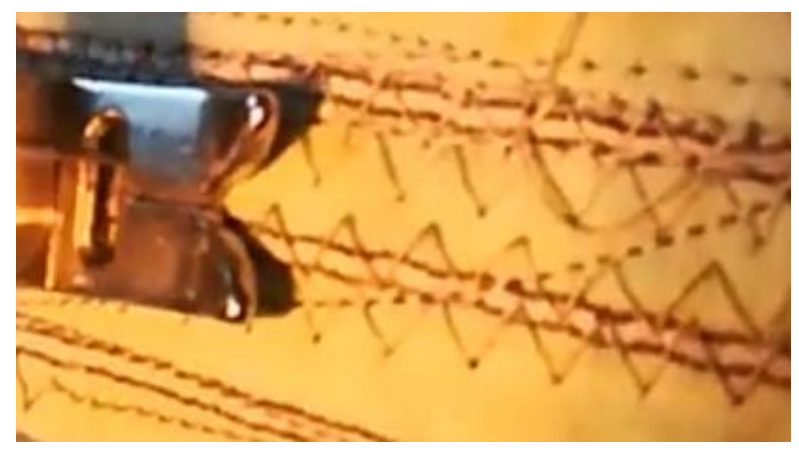

Figure 3. Zigzag stitch

The gear is shrunk onto the end of a short camshaft and is driven by a copper cogwheel. It could be replaced with a turned metal part, but this would result in loud operation [7]. The damaged gear and the connected camshaft can be seen in fig. 4. It is driven by the main shaft of the sewing machine through the copper part visible in fig. 5.

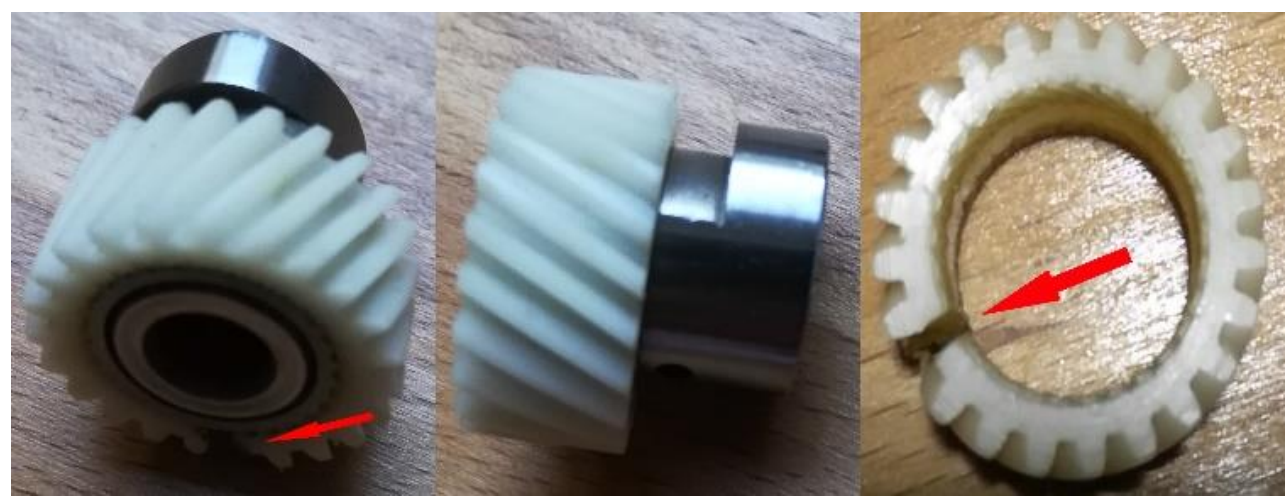

Figure 4. Broken section (shown by arrows)

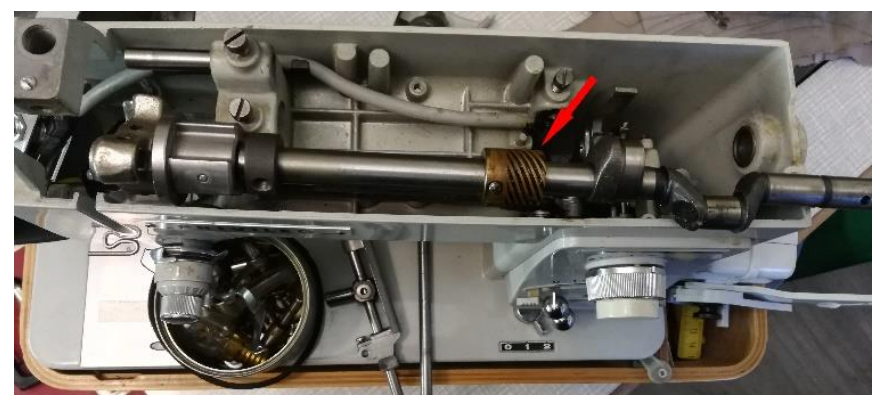

Figure 5. Disassembled sewing machine. Copper gear can be seen on the right end of the shaft. 
During the modeling process several challenges were faced, such as the helical gearing which had to be measured without a measuring machine, and it had to be decided whether to create the camshaft and the gear as one piece, or to keep the original camshaft and try to shrink the gear onto it. Since the bearing capacity of the used plastic was low, I finally decided to attempt the latter.

The part was printed with a Zortrax M200 3D printer by favour of BME, Department of Railway Vehicles and Vehicle System Analysis. The chosen material is ABS. In fig. 6, a polygonal section can be seen instead of a circular one due to an error in the settings of the CAD software, which carried on the final part.

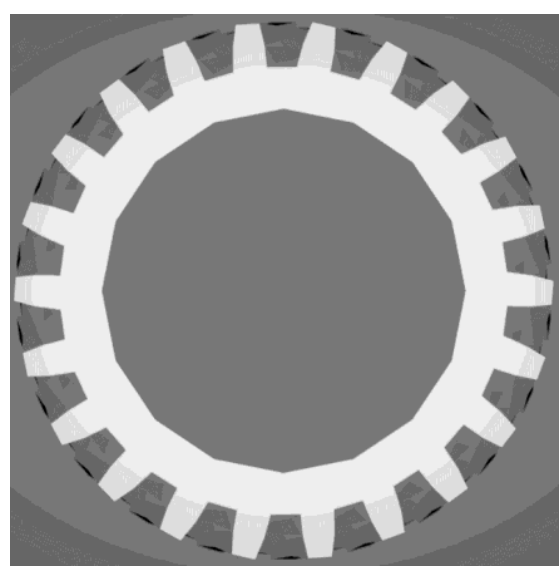

Figure 6. Polygonal bore profile

The following figures show the printer settings and the printing process.

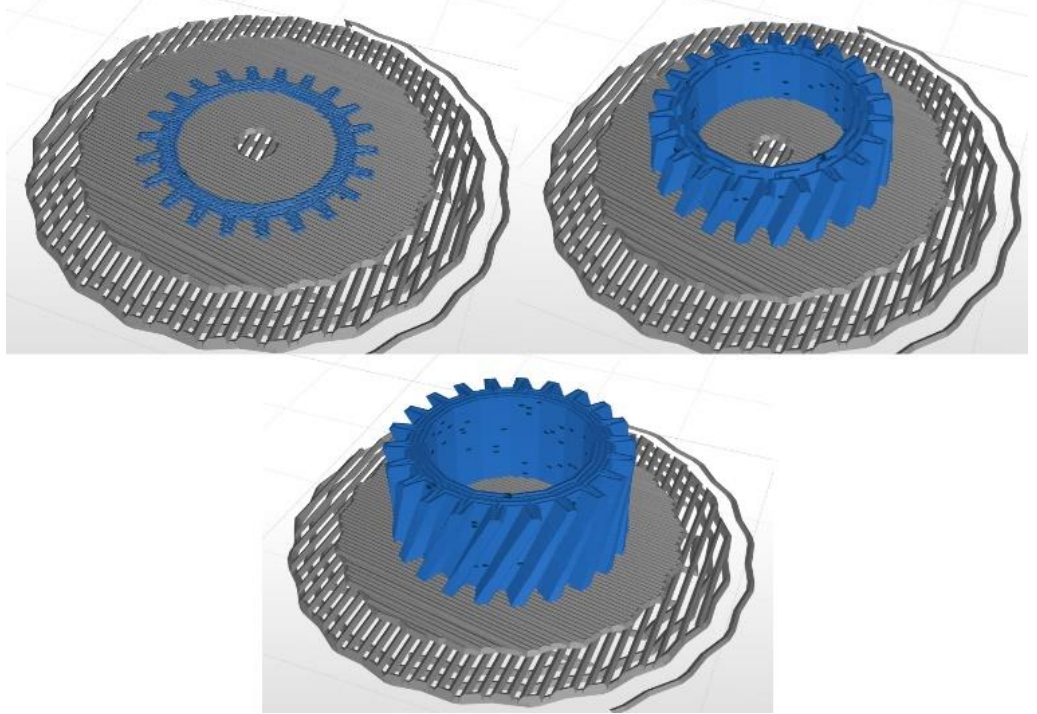

Figure 7. Printing process as shown by the slicing software 


\begin{tabular}{|l|l|}
\hline Support & $30^{\circ}$ \\
\hline Material & Z-ABS \\
\hline Layer height & $0.09 \mathrm{~mm}$ \\
\hline Infill & $20 \%$ \\
\hline Top surface layers & 9 \\
\hline Bottom surface layers & 4 \\
\hline Raft layers & 6 \\
\hline Printing speed & $50 \mathrm{~mm} / \mathrm{s}$ \\
\hline
\end{tabular}

Table 1. Printer settings.

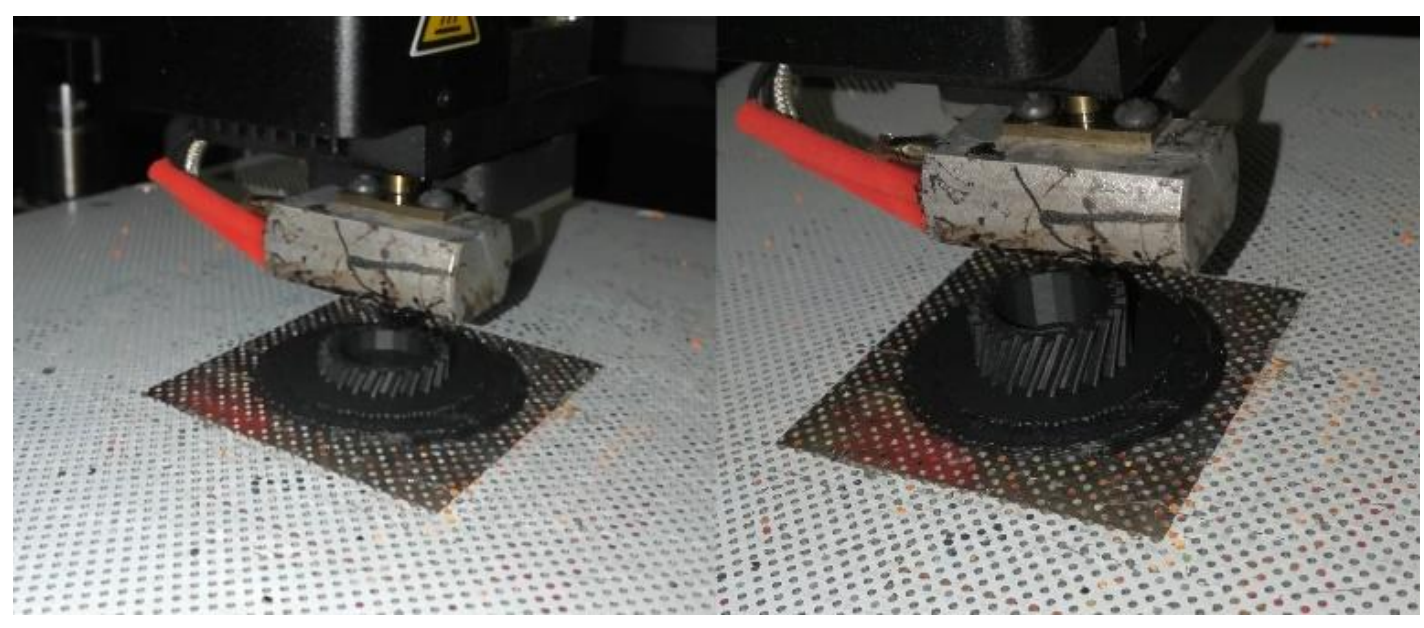

Figure 8. The printing process.

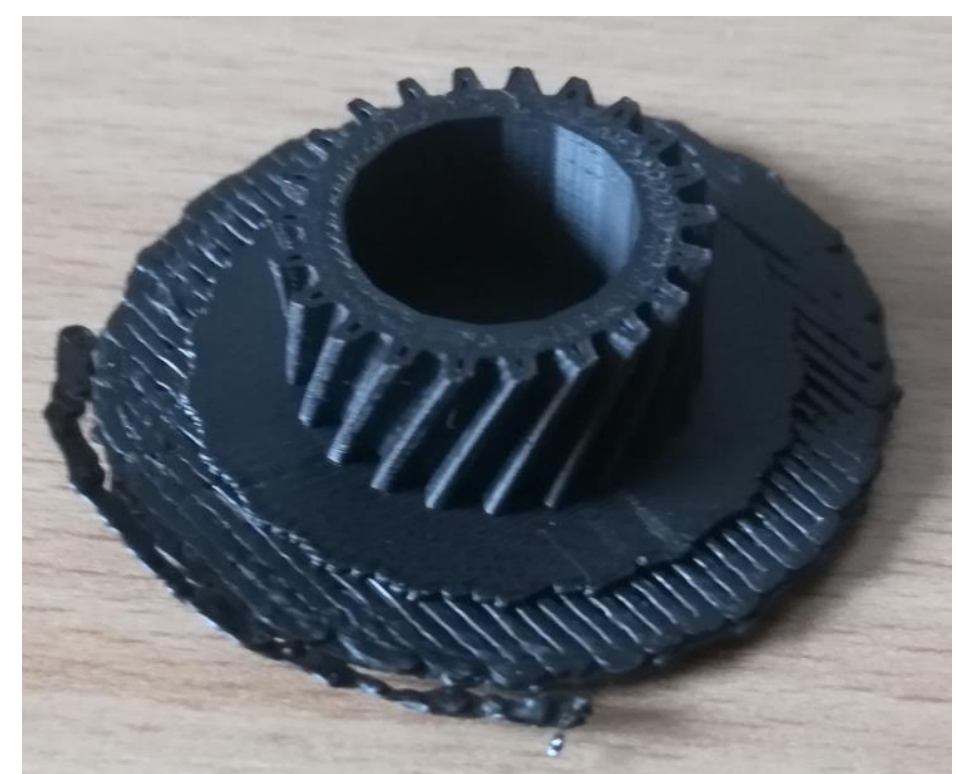

Figure 9. Finished part attached to the raft. 
In fig. 10, a comparison of the original and the reverse-engineered gearing can be seen. The width of the cogs and the distance between them is not perfect, but the angle of the helical gearing is close to the original.

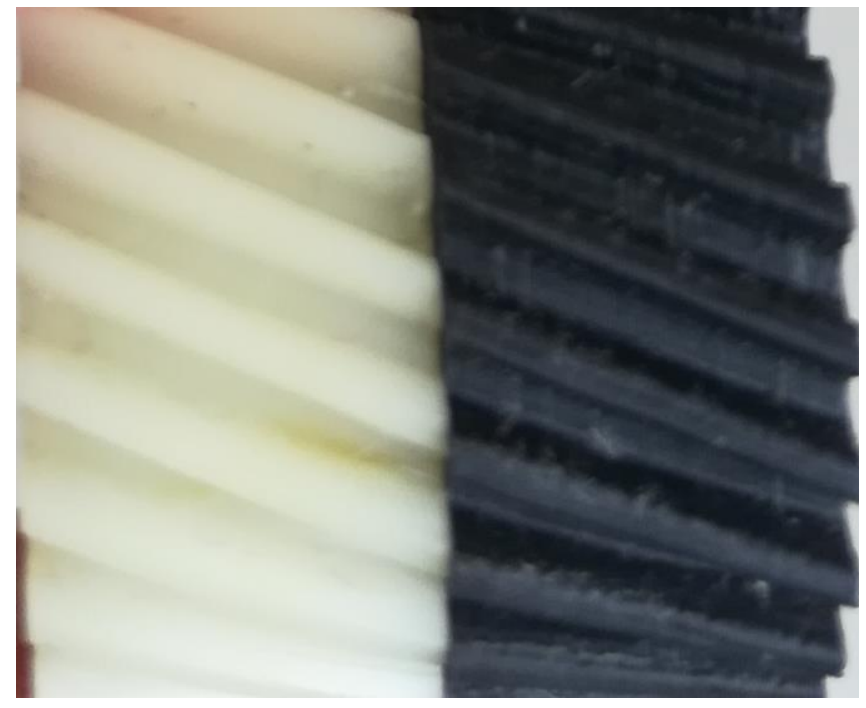

Figure 10. Comparison of the two gears.

Unfortunately, the piece was damaged while attempting to shrink it onto the camshaft (see fig. 11) - this was due to a difference in the size of the bore. Before printing a second prototype has to be optimized [8], the polygonality of the circles was fixed and the size of the bore was increased. This new piece was printed by a Creality Ender 3 v2 3D printer. The new piece can be seen on fig. 12 .

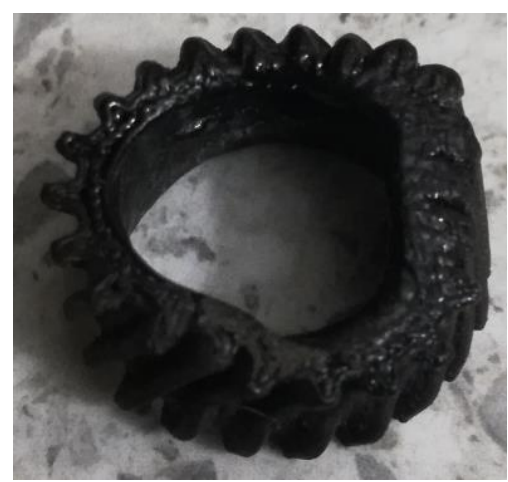

Figure 11. Damaged gear.

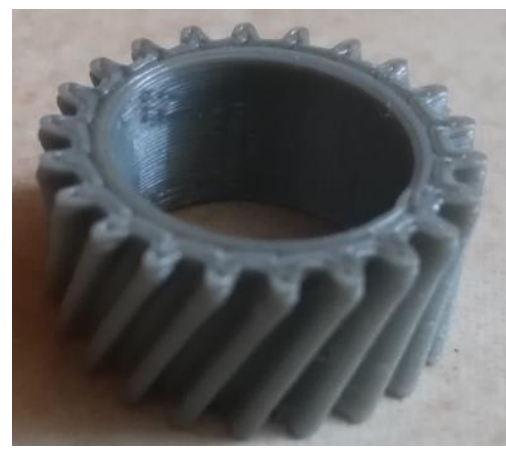

Figure 12. New gear.

Although the utilized material was PLA, the infill was set to $100 \%$, and the result was much closer to the original thanks to the improved 3D model [9]. The camshaft was cooled down to $-10{ }^{\circ} \mathrm{C}$, the gear was 
greased with soap and gently jiggled onto it. Fortunately, there was no rotation between the two parts, so it was ready to install. The assembled part can be seen in fig. 13.

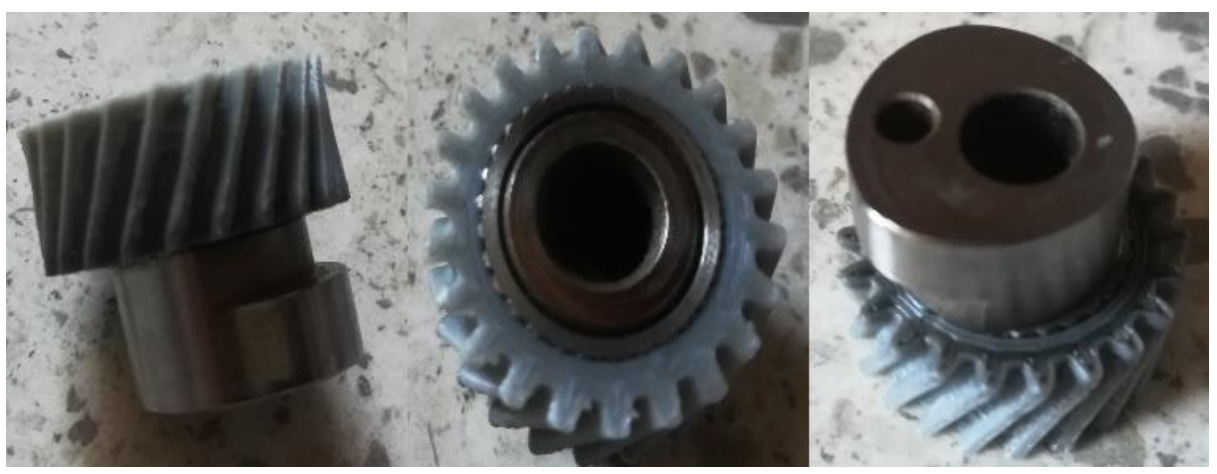

Figure 13. Assembled part, ready to install.

\section{Results and discussion}

The finished workpiece was installed into the sewing machine. The result can be seen in fig. 14 and in the video accessible by clicking the links on page 5 . Since the first model performed its task there was no need for further modeling and production. The only question left is the longevity, which will be revealed by the daily usage of the machine.

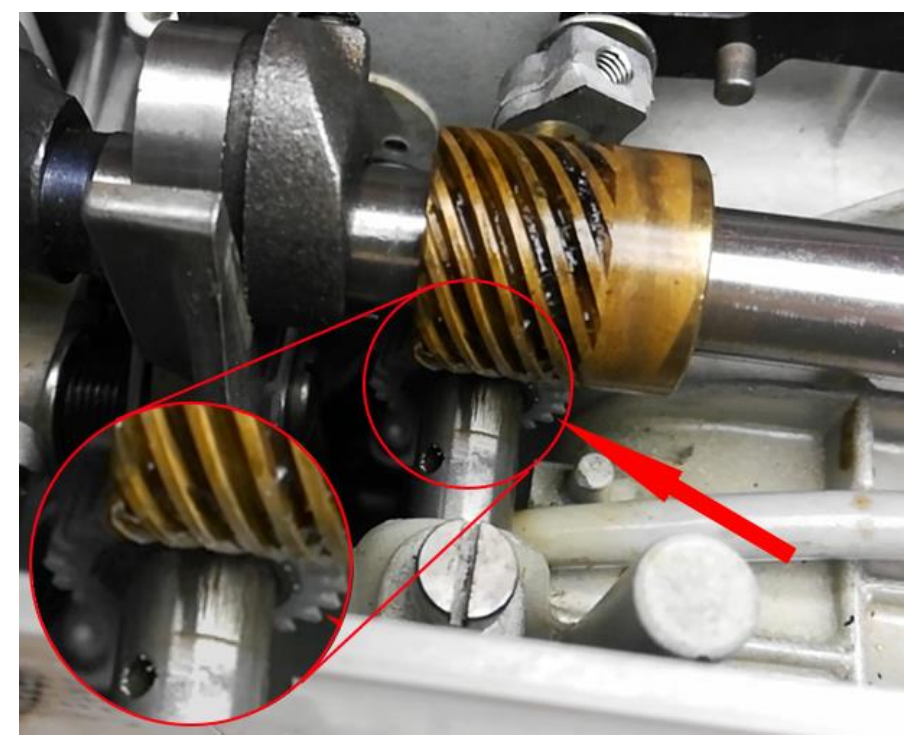

Figure 14. The part installed into the sewing machine.

\section{Summary and conclusion}

In conclusion, the experiment shows that with relatively little work and money investment, fully functioning parts can be manufactured using even entry level 3D printers and basic CAD modeling knowledge. Neither expensive, complicated measuring techniques and cutting machines, nor qualified technicians were employed, which would have significantly raised the cost to produce the presented workpiece. Beyond that, 3D printers can be used for various other projects, whether it be everyday 
objects of use, parts, or trinkets. Based on the project it can be stated that 3D printing really is the technology of the future.

\section{Links}

Rotation of the new gear - $\underline{\text { https://youtu.be/dDTeS8QHTbE }}$

Sewing machine in action - https://youtu.be/pU31GzpBKl8

\section{References}

[1] Khosrow-Pour, M. (2018) 'Encyclopedia of Information Science and Technology', IGI Global Information Science Reference, Hershey, USA

[2] Candi, M., Beltagui, A. (2018) 'Effective use of 3D printing in the innovation process', Technovation, 80-80, pp. 63-73, doi: 10.1016/j.technovation.2018.05.002

[3] Chmielarz, G. (2019) 'Present state and future application of smart technologies in manufacturing processes' Production Engineering Archives, 24. pp. 14-19

[4] Gebhardt, A. (2013) '3D printing and its applications', RTe Journal, 10, URN: urn:nbn:de:0009-235626

[5] Lee, J. H., Singer, J. P., Thomas, E. L. (2012) 'Micro-/nanostructured mechanical metamaterials' Advanced materials, 24(36), 4782-4810, doi: 10.1002/adma.201201644

[6] Ficzere, P. (2020) 'Experimental Dynamical Analysis and Numerical Simulation of the Material Properties of Parts Made by Fused Deposition Modelling Technologies', Periodica PolytechnicaTransportation Engineering, 48:3, pp. 221-225., doi: 10.3311/PPtr.13947

[7] Macek, W., Branco, R., Szala, M., Marciniak, Z., Ulewicz, R., Sczygiol, N., \& Kardasz, P. (2020). 'Profile and Areal Surface Parameters for Fatigue Fracture Characterisation', Materials, 13(17), 3691.

[8] Mankovits, T., Szabó, T., Kocsis, I., \& Páczelt, I. (2014). Optimization of the shape of axi-symmetric rubber bumpers. Strojniški vestnik-Journal of Mechanical Engineering, 60(1), 61-71

[9] Ulewicz, R., Nový, F. (2019). 'Quality management systems in special processes', Transportation Research Procedia, 40, 113-118. 